\title{
Introduction to Scientific Visualization
}

\author{
Edward Angel \\ Departments of Computer Science and Electrical and Computer Engineering \\ University of New Mexico \\ Albuquerque, NM 87131 USA \\ angel@cs.unm.edu
}

\begin{abstract}
As the power of computers has increased, scientists and mathematicians are having increasing difficult in interpreting the results of their simulations and computations. In addition, the volume of data that is generated defies our ability to store and interpret our results. Scientific visualization combines the power of the human visual system with the power of modern computer graphics to display large data sets in a fashion that can lead to both understanding and insight. In this presentation, we shall introduce the basic ideas behind this new field. First, we shall discuss the basic notions of modern computer graphics and how computer graphics can be used to display nongeometric data. We then examine two fundamental applications: scalar field visualization and vector field visualization. We will also examine some of the available software systems. Finally, we will examine recent work in scientific visualization on high-performance computers.
\end{abstract}

\section{Introduction:}

Scientific visualization is the field that combines data with computer graphics to provide insight into mathematics, science, engineering, and other fields through the power of the human visual system. The field has flourished over the past few years due to the incredible increases in computing power that are available at a reasonable cost.

Computer graphics deals primarily with geometric objects, such as lines, polygons, curves, and surfaces, that exist in three-dimensional space. At present, low-cost commodity graphics cards costing a few hundred dollars can render geometric objects with shaded, lit, and texture mapped at speeds measured in the millions of polygons/second. At the same time, our ability to run large-scale simulations has led to massive data sets that can be measured in terabytes. Such data sets present a multitude of difficulties, ranging from difficulties in storing them for later access to our inability to examine each datum. The appeal of scientific visualization is that if we can use the power of the modern graphics system to display the data, then we can use the power of the human visual system to detect the information in the data. However, we must first convert the data, which are generally simply numbers, to geometric objects that can be displayed. In this presentation, we shall see that there are many ways to convert a given data set and much of the creativity in scientific visualization comes from identifying new ways to associate data with geometry.

We start the presentation by reviewing the standard graphics model and show how it can be used for visualization. Then we examine the visualization of scalar fields such as describe implicit functions and data from medical technologies, including CT and MRI. We then turn to visualization of vector fields, concentrating on visualization of three-dimensional flow. Next, we examine the hardware and software issues. We shall discuss some of the available toolkits. On the hardware side, we concentrate on recent work in visualization on clusters.

\section{Computer Graphics and Visualization}

The basic paradigm behind all modern computer graphics is known as the synthetic camera model [1]. It is based on the idea that forming an image using a computer is similar to how we form an image by most standard imaging systems, such as cameras and telescopes. That is we combine the independent specifications of the objects and the camera through the mathematical process of projection. Most graphics systems are based on implementing this paradigm either through ray tracing or through a pipeline renderer. Although the ray tracer, which simulates the propagation of light from sources until it enters the lens of a virtual camera, is closer to how images are formed physically, ray tracers are slower than pipeline renderers and are not suited for real-time applications. 
A typical rendering pipeline is pictured in Figure 1. The geometry is specified through a set of points in space (vertices) that define polygons, curves, and surfaces. The image is eventually formed in a set of discrete picture elements or pixels in a frame buffer. Because most graphics systems are designed to render polygons, particularly triangles, typically higher order entities are decomposed into triangles by the software, or in the case of many visualization algorithms, only triangles are produced. All the geometry passes through affine transformations that convert the geometric data into the proper coordinates for the camera. As polygons pass through the pipeline, the renderer keeps track of the closest opaque pixels so that only the visible objects are displayed. For visible objects their colors are computed based on the material properties assigned to them and on the lights in the scene. Finally, the three-dimensional data is projected to the two-dimensional viewing surface and optionally textures can be mapped to the image.

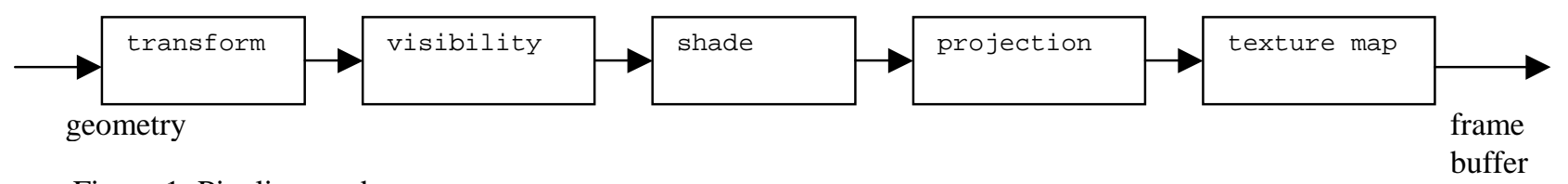

Figure 1: Pipeline renderer

All the blocks in the pipeline rendering process can be implemented in hardware and that accounts for the dominance of this paradigm in graphics and visualization systems. For applications in scientific visualization, we can decide on what type of geometric objects to use, how to shade them, and whether to use effects such as texture mapping.

\section{Scalar Field Visualization}

Consider a scalar valued function, $\mathrm{F}(\mathrm{x}, \mathrm{y}, \mathrm{z})$, in three-dimensions. The function may be known analytically or values may be known as a set of samples, $\left\{F\left(x_{i}, y_{j}, z_{k}\right)\right\}$ over a grid. The latter version arises in many simulation applications where the samples of $\mathrm{F}$ are values of physical variables that come out of the solution of partial differential equations. Medical imaging systems such as MRI and CT generate data of this form, usually over a regular grid.

The first form of the problem arises in understanding implicit functions of the form

$F(x, y, z)=0$,

where we assume that $\mathrm{F}()$ is known analytically. The function can describe no, one, or more isosurfaces, but there is no algorithm that gives us a set of uniformly distributed points on the surface(s) that we can use to generate a mesh we can display. The usually way of finding a set of samples on the surface(s) is to use an indirect method by converting the problem to second one by sampling F over a grid. Hence, we concentrate on the sampled version of the problem. We shall also consider only problems on a regular three-dimensional grid. Thus, we start with an array of volume elements, or voxels, each of which has a scalar value. The scalar value may represent a point sample at the center of the voxel or a weighted average of the scalar field over the voxel.

There are two general categories of scalar field methods: isosurface methods and direct rendering methods. In direct rendering methods, every voxel may contribute to the image. There is a variety of direct approaches, but all rely on the idea that we sum the contributions of voxels along rays from the viewer as in Figure 2. Each voxel is assigned a color and a transparency that can be directly related to a physical variable or be determined by what we wish to see. For example, in medical data, if we want to see the inside of the skull, we can make all the voxels corresponding to bone transparent. There are at least three strategies commonly used in direct rendering. In splatting [2], the voxels are composited in a back-to-front manner. If we consider the scalar value of each voxel to be an ideal point sample from a continuous scalar field, we can apply sampling theory determine how to form the image. Because the ideal reconstruction filter is not realizable, we can use well-understood approximations 
such as Gaussians. Because each voxel has the same shape, each projected reconstruction function is identical and has an identical two-dimensional shape, called its footprint. We can use two-dimensional bitmaps for the footprints, thus avoiding all three-dimensional calculations, such as projection. We need only find where the center of the footprint is in the image. Consequently, if the data are spatial organized so that a back-to-front compositing can be done easily, splatters are very fast. However, because there is essentially no threedimensional component to the process, the resulting image looks somewhat flat as in Figure $\mathrm{x}$.

In volume ray tracing [3], an image is produced as in a conventional ray tracer, treating each voxel as a geometric object. In texture-based direct rendering, the voxel array is used a three-dimensional texture. Each of these techniques has its own strengths and weaknesses. One of the major problems with ray tracing and splatting is that they are image-based and a new image must be computed for any change in viewing conditions. Even simple ray tracers need access to the entire data set for the computation. Consequently, for large data sets, ray tracers are best implemented on shared-memory architectures and do not map well to commodity clusters. Figure x shows two ray cast images from the Visible Woman data set that were produced at the University of Utah. The differences in the image are due only to the different colors and opacities assigned to the materials.

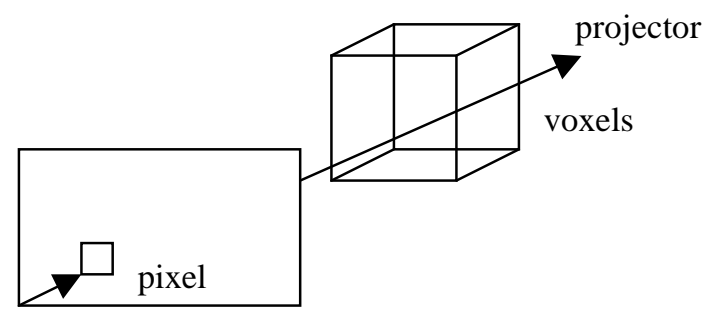

Figure 2. Direct volume rendering

Texture mapping provides an interesting hardware-based technique. As we saw in Figure 1, textures can be applied to primitives at the end of the pipeline. The most common form of a texture map is a two-dimensional image that is stretched to fit surface as the surface is rendered. Usually this process is done in hardware and produces an image of apparent complexity even though the geometry may remain simple. Texture mapping has proven so successful that even low-cost commodity graphics cards now include a fair amount of texture memory.

Three-dimensional textures are a natural extension. Such a texture is defined by a three-dimensional array $\mathrm{T}_{i j \mathrm{j}}$. Geometric surfaces can be thought of as surfaces through the texture space and thus each $x, y, z$ point on the surface has a corresponding point in the texture array that determines its color and transparency. A simple method for using three-dimensional textures for visualization is shown in Figure 3. The cube is represents a threedimensional texture that has been loaded into memory. We can cut through the cube with a series of parallel quadrilaterals, such as the one shown in the Figure 3. The texture is mapped onto each of the quadrilaterals and they are projected as another geometric entities. We can usually get a reasonable quality display using only a few hundred quadrilaterals rather than the tens of thousands of triangles that might be generated by methods such as marching cubes. With hardware texture mapping and sufficient texture memory, we can use this method at interactive rates.

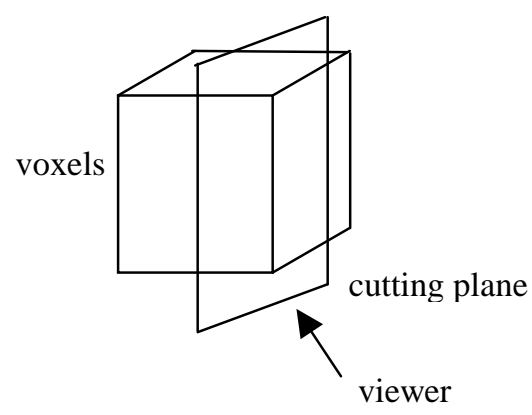


Figure 3. Visualization with three-dimensional textures

Isosurface techniques seek to display only those voxels that satisfy the relationship

$\mathrm{F}(\mathrm{x}, \mathrm{y}, \mathrm{z})=\mathrm{C}$,

where $\mathrm{C}$ is the desired isosurface value. These values form one or more surfaces, However, because we have only samples of F, we seek an approximate surface. The most popular method is marching cubes [4], which is based in the formation of cells from groups of 8 adjacent voxels. The vertices of the cells can be labeled black or white depending on whether the value of the corresponding voxel is greater than or less than the isosurface value. There are 256 possible binary color codings of the cube. However, if we account for rotational symmetries and the symmetry between black and white, we can reduce these to 14 distinct cases. Unless all the vertices are colored the same, at least one piece of the isosurface must pass through the cell and can be approximated by triangles, as illustrated in Figure 4.

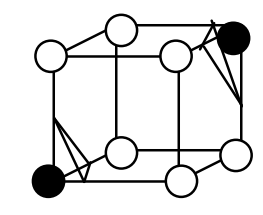

Figure 4. Cell with two triangular pieces of isosurface

Marching cubes has some important advantages which account for it popularity. It produces geometric objects, triangles, which can be displayed interactively on any graphics system and it is inherently parallel, as each cell generates triangles independently of all the other cells. In addition, we can encode each pattern into an index and make the algorithm table-driven.

In spite of its popularity, marching cubes has some deficiencies. First, because it is an isosurface method, not all voxels contribute to the image and thus we may not image some important information. Second, the number of triangles generated depends on the sampling rate and not on how many are needed to display the isosurface. A second process, triangle decimation, can be used to reduce the triangle count but often the resulting mesh is not smooth.

An alternative isosurface method is to use a particle system to find locations on the surface [5]. The particles are programmed to have a behavior that seeks an isosurface in the data array. Those particles which do not find an isosurface are killed off while those that do are allowed to split. Particles repel each other so they distribute themselves over the surface and can be controlled so that there is a greater density of particles in regions where there is a small radius of curvature. Figure 6 shows an isosurface computed my marching cubes and a mesh generated by a particle system in top.

\section{Flow Visualization}

In vector visualization, the function $F$ is vector valued. The most common example is flow visualization where we have a velocity at each spatial point. Often we also have ancillary data such as pressure and temperature in addition to velocity. Once more, we will assume the data are on a regular grid.

One of the simplest methods to display flow data is through the use of icons which can be placed within a threedimensional space and projected to form an image. We can generate arrows or lines pointing in the direction of the flow and whose length is proportional to the magnitude of the velocity. Usually, to avoid visual clutter there is some sort of sampling process so that only a subset of the data is displayed. For example, we can look at a grid of locations and only display those whose magnitude exceeds a predetermined threshold. Figure $\mathrm{x}$ shows a flow visualization of currents in the oceans in which cones are used to indicate the direction of the flow. The size of each cone is proportional to the flow magnitude and the color of the cone is determined by the temperature. The continental shelf was determined using an isosurface method.

Generally, we wish to display the path weightless particles would follow in the flow. For steady-state flow, these paths are streamlines or particle traces [6]. They can be formed by starting somewhere in the data set, typically where the magnitude of the velocity exceeds some threshold value, and using the velocity values to integrate a path through the data set until the magnitude goes below threshold or the path returns to the starting point or 
leaves the region where we have data. We can display streamlines using another variable, such as temperature, to color the streamline for display. We can also group streamlines into streamtubes to get a better visualization of how the flow spreads out or contracts as in Figure 7.

Although streamline-related methods dominate flow visualization, there are other techniques. Often simply coloring each data point by the magnitude of the velocity is sufficient to display the data set (Figure 8) [7]. One of the difficulties with streamline methods is to display sufficient streamlines to see the information in the data set while not cluttering the display to the point that the viewer misses significant information. Recently, line integral convolution (LIC) [8] has received considerable interest. In this method, the velocity data are convolved with a random mask and integrated along the flow direction. All data points contributed to the image while the convolution gives sufficient correlation for the viewer to detect local features.

\section{Parallelization}

As computers have become more powerful so have our visualization techniques. However, our methods are constantly challenged by the size of the data sets that are generated by simulations, especially using high performance computers. Many visualization researchers are investigating methods that can take advantage of parallel computers, especially clusters of commodity computers equipped with commodity graphics cards.

Algorithms for these architectures have been generally divided into three groups [9]: sort first, sort last, sort middle. These designations arise from the how the rendering pipeline from Figure 1 can be implemented on with a collection of processors. Suppose that we have a collection of geometry units that carry out the transformations, shading, and projection steps, and a collection of raster units that can produce pixels from the vertices and do operations such as texture mapping. The main distinction in these architectures is where we do the sort, the step that is responsible for determining which object is in front. Figure 5 illustrates sort first and sort last.
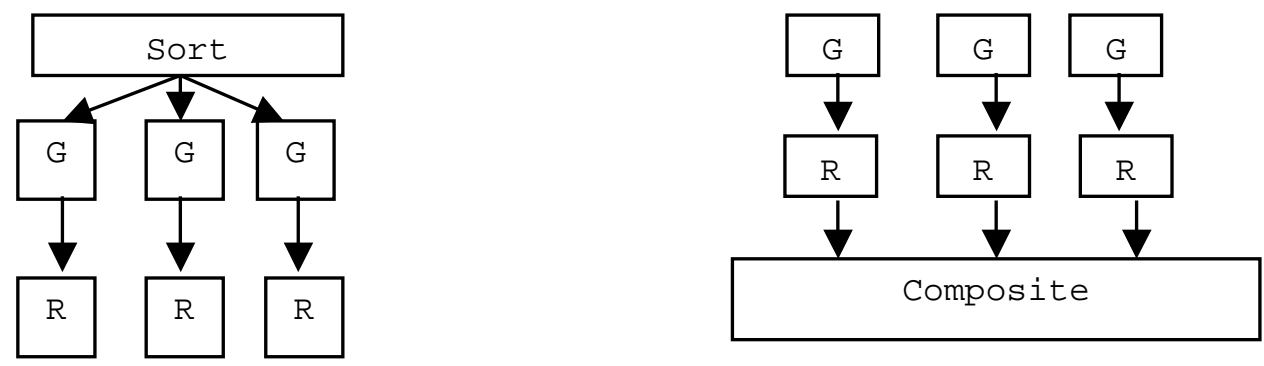

Figure 5. Sort-first and sort-last rendering.

In sort first, each raster unit is responsible for an area of the screen and gets its input from a corresponding geometric processor. However, first we must decide which parts of the data will be mapped to which parts of the image. Sort-first methods are particularly well suited for high-resolution displays such as power walls [10]. In many applications, there is a simple spatial decomposition that makes this possible. In sort-last rendering, data is distributed randomly to pairs of geometric and raster units. Thus, each raster unit has to deal with the resolution of the image. At the end, a compositing step is required to combine all the partial images. Although sort-last algorithms are simple to implement and achieve load balancing among processors, the final composite can be costly, especially for high-resolution images.

Another area of recent interest is high-resolution displays. Display technology has moved much slower that other areas such processing speed and memory density. CRT displays most LCD panels are still in the megapixel range. As data sets have become larger, these display do not provide sufficient resolution to display information in the data. A recent alternative has been to use arrays of digital projectors to produce high-resolution displays [11]. In terms of the sort-first sort-last classifications, sort-last is at a distinct disadvantage for these displays as each raster unit must maintain a frame buffer with the resolution of the whole display and large amounts of data must be moved around in the compositing step. On the other hand, sort-first works well because each raster unit needs a frame buffer whose size matches its part of the display. 


\section{Software}

There are three basic approaches to visualization software. On one end are packages that provide at least one method for each major problem but are inflexible. At the other extreme, there is the "do it yourself" approach where users write complete programs. In the middle are approaches which give the user a toolkit, such as VTK, or set of building blocks that the user can put together interactively, such as AVS or Khoros.

One of the major thrusts in visualization software research is in parallelism and distributed software. Not only is there the desire to use parallel methods within the visualization algorithms, but in addition large data sets cannot be transferred easily to remote users. Consequently, we often want to do our simulation and part of our visualization in one place, but see the results in another. Packages such as Khoros allow modules to be run on different computers in a network. Others such as VTK are incorporating parallelism with the modules.

\section{References}

[1] Angel, E., Interactive Computer Graphics (Second Edition), Addison-Wesley, 2000.

[2] Westover, L., "Footprint Evaluation for Volume Rendering", Computer Graphics, 24(4), 367-376, 1990.

[3] Levoy, M., "Display of Surfaces from Volume Data," IEEE Computer Graphics and Applications, 8(3), 2937, 1988.

[4] Lorensen, W.E., and H.E. Cline, Marching Cubes, A High-Resolution 3D Surface Construction Algorithm, Computer Graphics, 21(4), 163-169, 1987.

[5] Crossno, P.J., and E. Angel, "Isosurface Extraction Using Particle Systems", IEEE Visualization, Nov 1997.

[6] Schroeder, W., Martin K., and Lorensen, B, The Visualization Toolkit 2nd Edition, Prentice Hall.

[7] Johannsen, A. and R. Moorhead. "AGP: Ocean Model Flow Visualization," IEEE Computer Graphics and Applications, 15 (4): 28-33. July 1995.

[8] Cabral, B. and L. Leedom, "Imaging Vector Fields Using Line Integral Convolution," Computer Graphics 163-169. August 1993.

[9] Molnar, S., M. Cox, D. Ellsworth and H. Fuchs, A Sorting Classification of Parallel Rendering, IEEE Computer Graphics and Applications,July, 1994.

[10] Humphreys, G. and P. Hanrahan, A Distributed Graphics System for Large Tiled Displays, IEEE Visualization 1999.

[11] IEEE Computer Graphics and Applications, April, 2000.

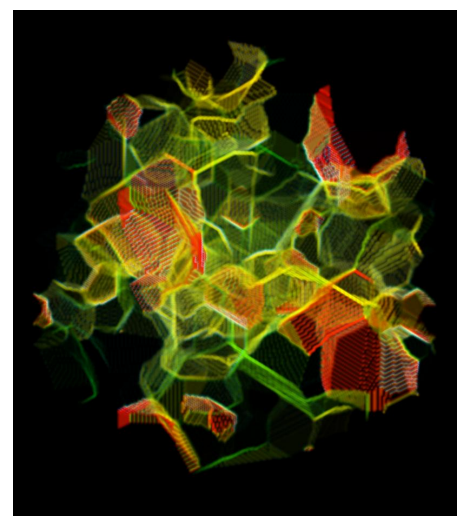

Figure 5. Splat showing material properties

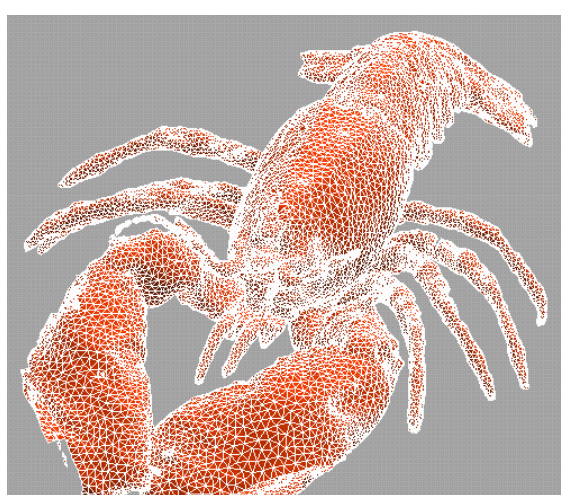

Figure 6: Isosurface by marching cubes with particle system superimposed 


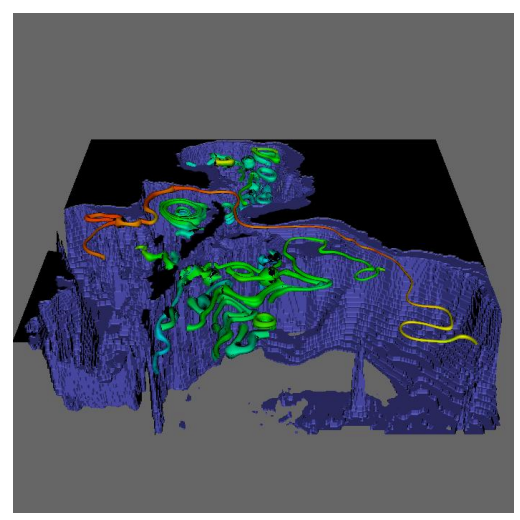

Figure 7. Streamtubes of ocean circulation

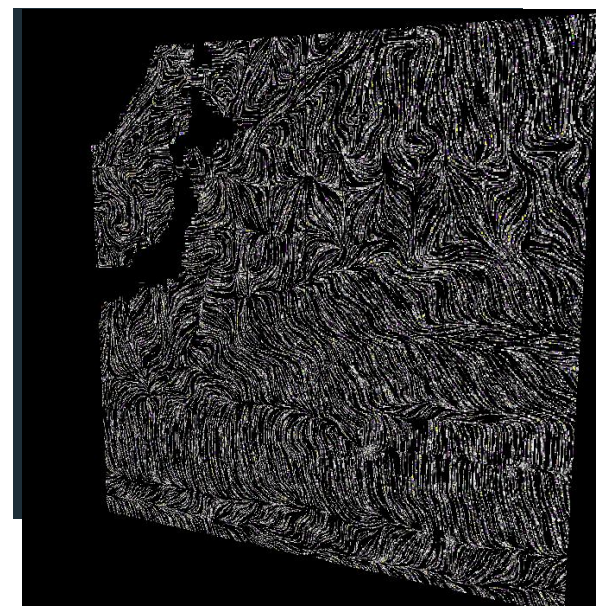

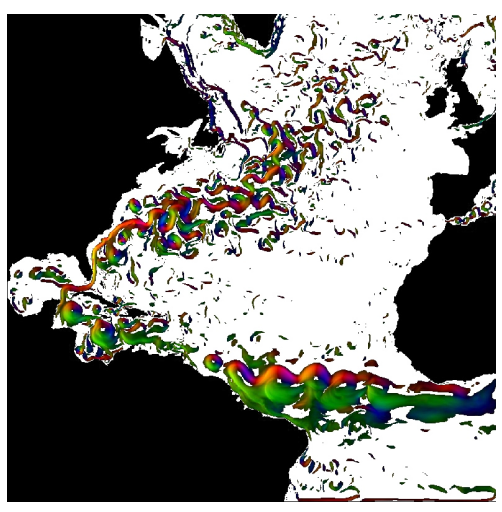

Figure 8. Color display of velocity data. 

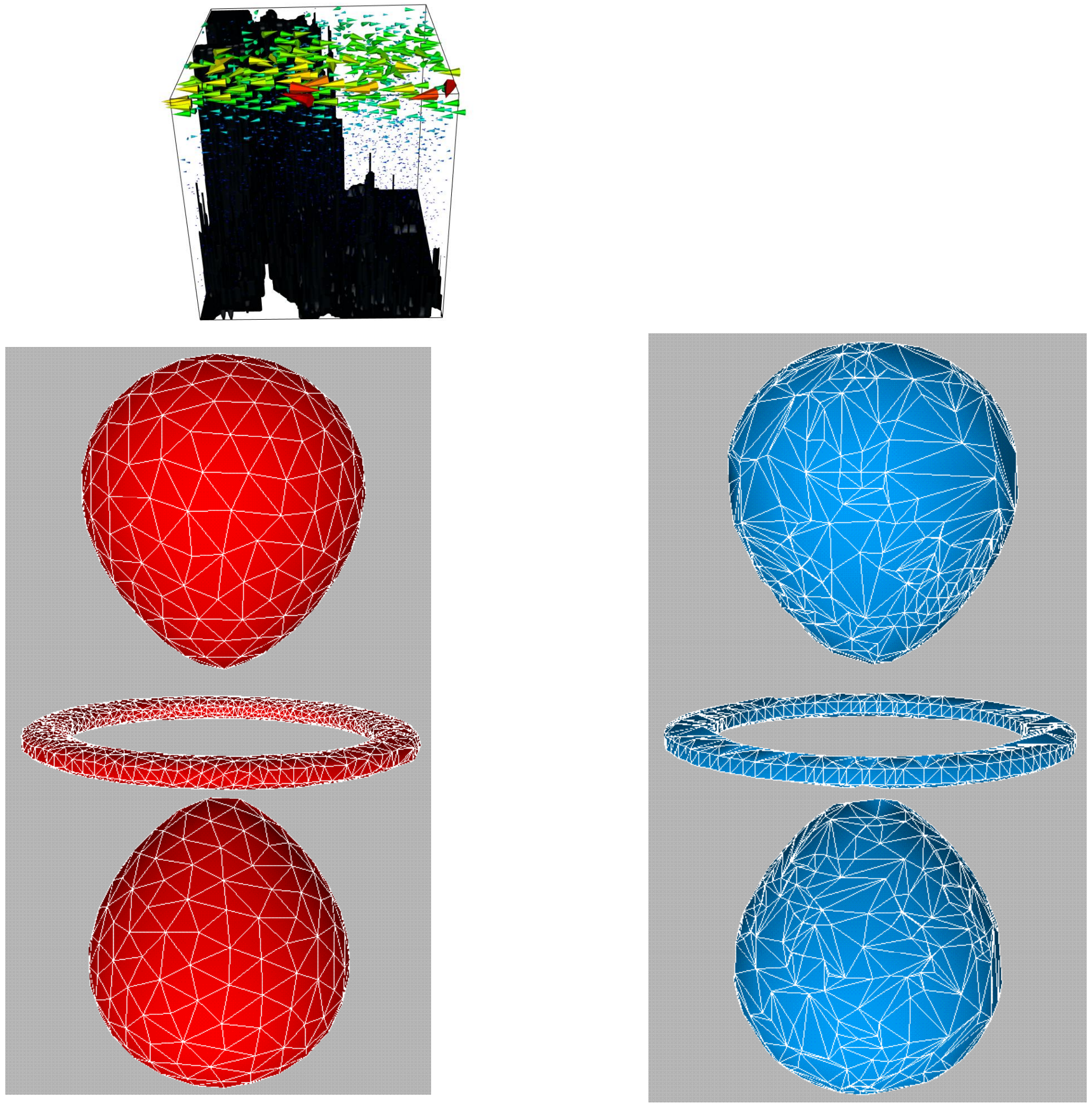
